\title{
Planar bilayer metamaterial with left-handed transmission and negative refraction at microwave frequencies
}

\author{
Atilla Özgür Çakmak ${ }^{*}{ }^{1,2}$, Kaan Guven ${ }^{1,3}$, and Ekmel Özbay ${ }^{1,2,3}$ \\ ${ }^{1}$ Nanotechnology Research Center, Bilkent University, 06800 Ankara, Turkey \\ ${ }^{2}$ Department of Electrical and Electronics Engineering, Bilkent University, 06800 Ankara, Turkey \\ ${ }^{3}$ Department of Physics, Bilkent University, 06800 Ankara, Turkey
}

Received 19 September 2006, revised 25 November 2006, accepted 6 December 2006

Published online 22 March 2007

PACS 41.20.Jb, 42.70.-a, 78.20.Ci, 81.05.Zx

A planar composite metamaterial consisting of bilayers of metal cutwire pairs and long wire pairs which are separated by a thin dielectric layer is designed and fabricated for microwave frequencies. The simulated and experimentally measured transmission spectra of the metamaterial and its individual components (cutwire-only and wire-only) indicates that the metamaterial exhibits a transmission band within the common stop bands of its components, and thus acts as a medium with negative index of refraction. The existence of $n<0$ is further supported by a refraction experiment.

(ㄷ) 2007 WILEY-VCH Verlag GmbH \& Co. KGaA, Weinheim

\section{Introduction}

Metamaterials are artificial structures with tailored electromagnetic response to the incident electromagnetic excitation at the desired frequency band. The unusual property of the metamaterials is that they can behave like a medium with negative effective index of refraction, $n_{\mathrm{eff}}<0$ [1-3]. Decades ago, it was predicted that such a medium would exhibit completely novel electromagnetic phenomena [4], yet no naturally occurring material had been known to exist to support the theories experimentally.

The first composite metamaterial (CMM) design consists of stacked dielectric layers on which planar metal wires and metal split ring resonator (SRR) arrays have been printed periodically [1]. The combined electric responses of the SRR and wire elements provide an effective plasmon cutoff frequency, $\omega_{\mathrm{p}}$, below which the permittivity, $\varepsilon(\omega)$, becomes negative. When the magnetic field component of the incident electromagnetic wave is normal to the SRR plane, the inductance of the rings and the capacitance at the gaps generate a resonance at a particular frequency, $\omega_{\mathrm{m}}$. For frequencies $\omega_{\mathrm{m}}<\omega$, the effective permeability, $\mu(\omega)$ becomes negative. Hence, the CMM may act as a medium with $\varepsilon(\omega)<0, \mu(\omega)<0$, for $\omega_{\mathrm{m}}<\omega<\omega_{\mathrm{p}}$. These materials are also called left-handed materials due to the axes formed by the electromagnetic field vectors. A one dimensional metamaterial can be constructed by stacking SRR and wire layers alternatingly. There are numerous studies which employ different SRR/wire patterns for metamaterials. Most of these designs adhere to the aforementioned SRR topology. In this case, the electromagnetic wave vector is required to be in the plane of metamaterial layers and significant number of layers should be stacked to provide sufficient thickness for the incident field. This requirement turned out to be a major drawback for the fabrication of the CMMs at infrared and optical frequencies [5-8].

Recently, a different topology for obtaining magnetic resonance is proposed and demonstrated [9]. The SRR is replaced by metal cutwire strips separated by a dielectric layer, like a parallel plate capacitor

\footnotetext{
* Corresponding author: e-mail: atilla@ee.bilkent.edu.tr, Phone: +90 312290 1971, Fax: +90 3122664579
} 


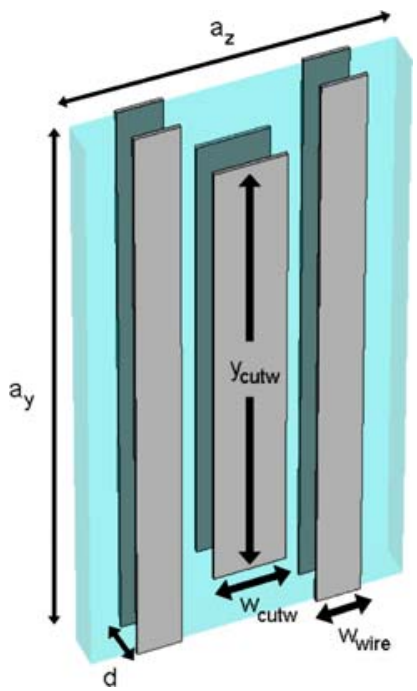

Fig. 1 (online colour at: www.pss-b.com) Schematic view of the bilayer metamaterial. The cutwires and wires are located on both sides of a thin dielectric layer, $d$. The unit cell contains one paired cutwire and two paired long wire elements. The geometrical parameters and periodicity of the structure are given in the text. The electromagnetic wave propagates in the $x$ direction with the electric field in the $y$ direction and the magnetic field in the $z$ direction. The bilayers are stacked in the $x$ direction with periodicity $a_{x}$

(see Fig. 1). The magnetic field parallel to the plane of metal strips can induce a resonance which leads to $\mu(\omega)<0$. This allows the electromagnetic field to propagate normal to the CMM plane, as opposed to the parallel propagation of the former SRR/wire topology. As a consequence of this idea, a number of new metamaterial studies have been reported [10-12].

In this paper, we employed this new topology to design, and fabricate a CMM for microwave frequencies. We measured the transmission spectra of the CMM and its cutwire and wire components separately, and demonstrated that the CMM exhibits a transmission band in the common stop bands of the cutwireonly and wire-only structures. A negative refraction experiment is performed which independently provides support for the existence of negative index of refraction of the CMM within the left-handed transmission band that is already obtained from the transmission spectrum. The paper is organized as follows: The CMM design and fabrication details are given in the next section. Third section describes the transmission and negative refraction experiments.

\section{Planar bilayer metamaterial design and fabrication}

The bilayer is fabricated from dielectric layers (thickness $d=0.4 \mathrm{~mm}$, dielectric constant $\varepsilon_{\mathrm{r}}=4.2$ ) with a metal coating (thickness $t=30 \mu \mathrm{m}$ ) on both sides. Figure 1 shows the schematic view of the CMM unit cell. $d$ is the thickness of the dielectric spacer between the pair elements. The cutwire dimensions are $w_{\text {cutw }}=0.8 \mathrm{~mm}, y_{\text {cutw }}=5.5 \mathrm{~mm}$, and the wire dimensions are $w_{\text {wire }}=0.5 \mathrm{~mm}, y_{\text {wire }}=7.0 \mathrm{~mm}$. The unit cell has dimensions $a_{x}=2.0 \mathrm{~mm}, a_{y}=7.0 \mathrm{~mm}, a_{z}=3.5 \mathrm{~mm} . a_{x}$ is the stacking periodicity in the propagation direction. With these parameters, the simulated cutwire structure exhibits a magnetic resonance around, $f_{\mathrm{m}} \approx 14 \mathrm{GHz}$. The wire units are designed with a high cut-off frequency in order to ensure the $\varepsilon<0$ within the $\mu<0$ stop band of cutwires. The wires are present on either side of the bilayer for symmetry.

\section{Transmission measurements}

The transmission spectra of the CMM, cutwire-only and wire-only structures were measured in free space, by a HP8510C Network Analyzer and a set of antennas. We employ a similar analysis conducted for SRR/wire type CMM in order to identify the left-handed transmission band [13]. Figure 2(a) and (b) summarize the measurement and simulation results, respectively. The $\omega_{\mathrm{p}}$ of the wire-only structure is very high, thus satisfying $\varepsilon(\omega)<0$ within the indicated stop band that spans the measured frequency range. The cutwire only structure exhibits a clear resonance gap around $14.25 \mathrm{GHz}$. In order to particularly identify this phenomenon, we used a complementary structure in which the cutwire pairs are elec- 

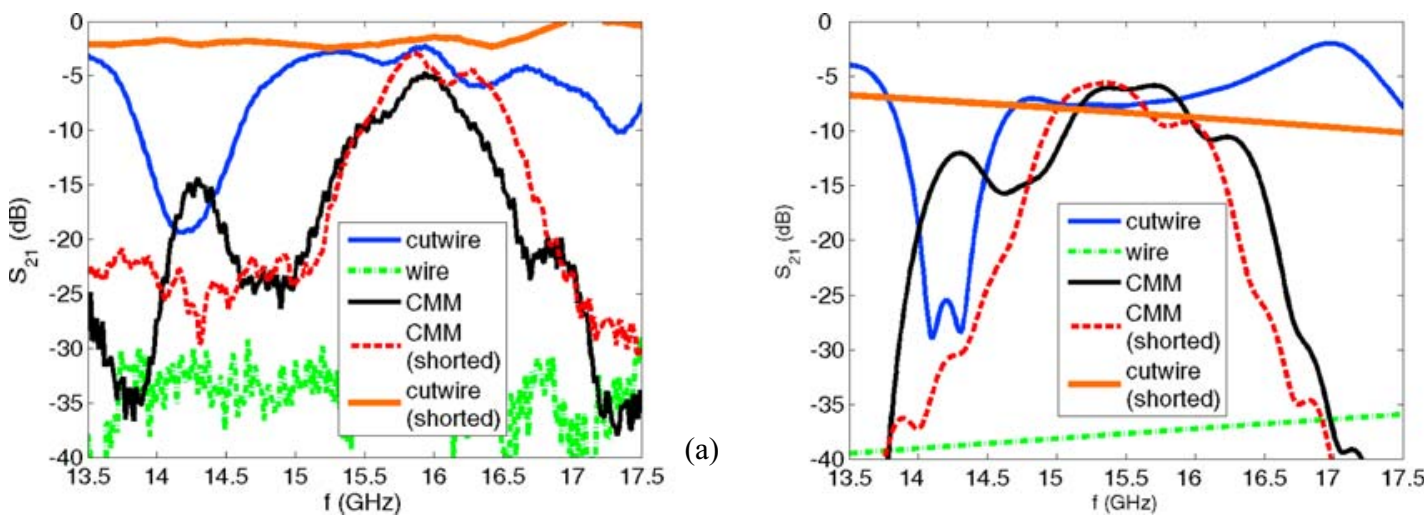

Fig. 2 (online colour at: www.pss-b.com) (a) Measured and (b) simulated transmission spectra of the CMM and its individual components. The left-handed transmission peak of the CMM around $14.25 \mathrm{GHz}$ appears in the magnetic resonance gap of the cutwires. The transmission band extending from 15.5 to $17 \mathrm{GHz}$ is right handed. Note that the electrically shorted cutwire pairs does not exhibit the magnetic resonance gap, which leads to the absence of the lefthanded peak for the shorted CMM.

trically shorted through the dielectric layer. Shorting the cutwire pairs removes the capacitive coupling of the cutwire pair, and therefore demolishes the resonance. Thus, the gap is guaranteed to be due to the magnetic resonance.

The CMM exhibits two distinct peaks. The transmission peak between 14.0-14.5 GHz coincides with the magnetic resonance of the cutwires. The shorted CMM which incorporates the shorted cutwire pairs does not show this peak. The transmission band between $15.5-16.5 \mathrm{GHz}$ is right handed, since it is common to both CMM and the shorted CMM. We note that the cutwires couple to the electric field as well, which has a dominating effect on the overall electric response of the CMM. Indeed, if the $\varepsilon(\omega)<0$ behaviour of the CMM were due to the wires only, the aforementioned right-handed peak should not be present.

The simulations are performed by commercial software (CST Microwave Studio ${ }^{\circledR}$ ). In the simulations, the structure is assumed to extend infinitely along the lateral directions. We anticipate that the finite size
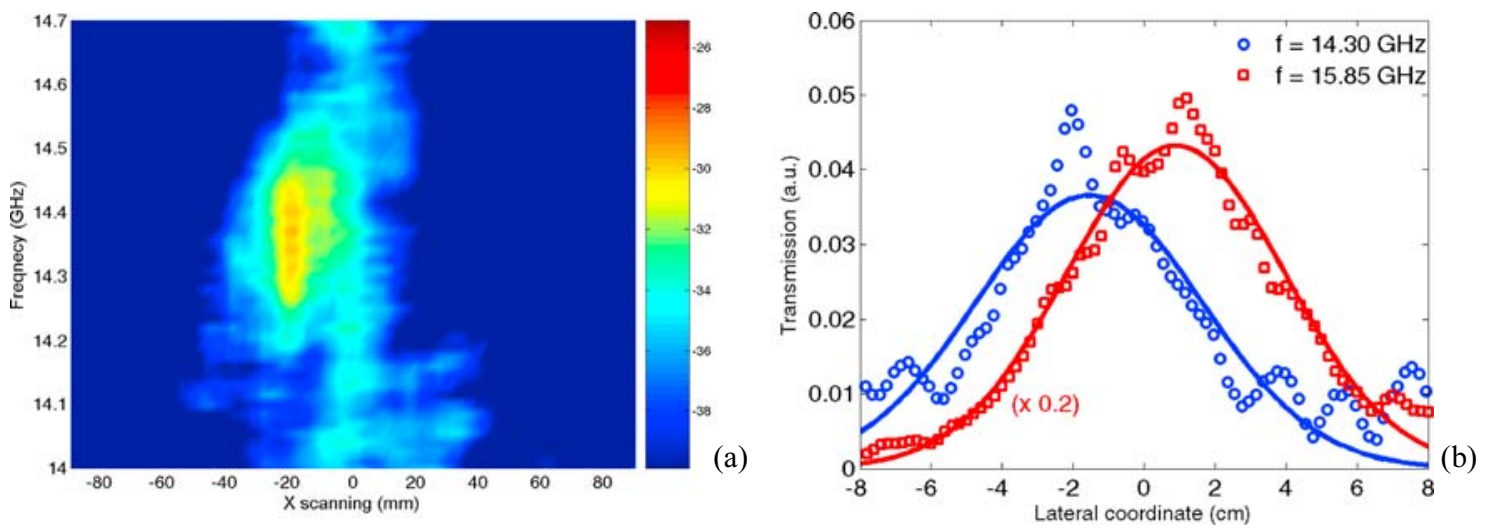

Fig. 3 (online colour at: www.pss-b.com) (a) Frequency vs. lateral spatial distribution of the transmitted signal at the CMM surface for $10^{\circ}$ incidence. Warm color indicates higher transmission. (b) Lateral power profiles taken at $f=14.30 \mathrm{GHz}$ and $f=15.85 \mathrm{GHz}$ from the left-handed and right-handed bands, respectively. The $f=15.85 \mathrm{GHz}$ profile is scaled to fit. The incident signal (not shown) is centred at the origin. 
of the fabricated structures in the $y$ direction may have an effect on the electric response of the CMM. Nevertheless, all the essential features of the experimentally observed spectra were obtained by simulations, with a good agreement in their respective spectral position.

\section{Negative refraction measurements}

In order to confirm that the CMM acts as a medium with $n_{\mathrm{eff}}<0$, we performed a negative refraction experiment using a bulk CMM structure consisting of 4 layers. The electromagnetic signal is incident at $10^{\circ}$ to the center of the CMM surface from the left side of the origin. The transmitted signal is scanned laterally along the exit surface of the CMM. Figure 3(a) shows the lateral and frequency distribution of the transmitted signal with color coded transmission level. Evidently, the beam transmitted within the left-handed band appears on the left side of the origin, indicating that the beam refracted negatively upon entering the CMM. The lateral power profile taken at $f=14.3 \mathrm{GHz}$ (within the left handed band) confirms the negative lateral shift of the beam. Figure 3(b) shows the lateral power profiles taken at $f=14.3 \mathrm{GHz}$ (within the left-handed band) and at $f=15.85 \mathrm{GHz}$ (within the right-handed band). Evidently, the lateral shift of the profiles agrees with the anticipated sign of the $n_{\text {eff }}$ for each transmission band.

\section{Conclusion}

In this work, we designed and fabricated a composite metamaterial for normal-to-plane propagation at microwave frequencies. The transmission properties of the CMM and its components are measured and simulated. The paired cutwire units are demonstrated to exhibit a magnetic resonance. A distinct lefthanded transmission band is observed for the CMM. The existence of a negative effective index of refraction for this band is confirmed by a refraction experiment. This type of metamaterial is very suitable for scaling to higher frequencies, due to the extremely compact design and easily adjustable thickness. This CMM topology may become feasible for integration in various microwave applications.

Acknowledgements This work is supported by the European Union under the projects EU-DALHM, EU-NOEMETAMORPHOSE, EU-NOE-PHOREMOST, and TUBITAK under projects No. 104E090, 105E066, 105A005. One of the authors (Ekmel Ozbay) also acknowledges partial support form the Turkish Academy of Sciences.

\section{References}

[1] D. R. Smith, W. J. Padilla, D. C. Vier, S. C. Nemat-Nasser, and S. Schultz, Phys. Rev. Lett. 84, 4184 (2000).

[2] R. A. Shelby, D. R. Smith, S. C. Nemat-Nasser, and S. Schultz, Appl. Phys. Lett. 78, 489 (2001).

[3] R. A. Shelby, D. R. Smith, and S. Schultz, Science 292, 77 (2001).

[4] V. G. Veselago, Sov. Phys. Usp. 10, 504 (1968).

[5] T. J. Yen, W. J. Padilla, N. Fang, D. C. Vier, D. R. Smith, J. B. Pendry, D. N. Basov, and X. Zhang, Science 303, 1494 (2004).

[6] S. Linden, C. Enkrich, M. Wegener, J. Zhou, T. Koschny, and C. M. Soukoulis, Science 306, 1351 (2004).

[7] C. Enkrich, M. Wegener, S. Linden, L. Zschiedrich, F. Schmidt, J. F. Zhou, T. Koschny, and C. M. Soukoulis, Phys. Rev. Lett. 95, 203901 (2005).

[8] H. O. Moser, B. D. F. Casse, O. Wilhelmi, and B. T. Saw, Phys. Rev. Lett. 94, 063901 (2005).

[9] V. M. Shalaev, W. Cai, U. K. Chettiar, Hsiao-Kuan Yuan, A. K. Sarychev, V. P. Drachev, and A. V. Kildishev, Opt. Lett. 30, 3356 (2005).

[10] G. Dolling, C. Enkrich, M. Wegener, J. F. Zhou, C. M. Soukoulis, and S. Linden, Opt. Lett. 30, 3198 (2005).

[11] J. Zhou, L. Zhang, G. Tuttle, T. Koschny, and C. M. Soukoulis, Phys. Rev. B 73, 041101 (2006).

[12] S. Zhang, W. Fan, N. C. Panoiu, K. J. Malloy, R. M. Osgood, and S. R. J. Brueck, Phys. Rev. Lett. 95, 137404 (2005).

[13] K. Aydin, K. Guven, M. Kafesaki, L. Zhang, C. M. Soukoulis, and E. Ozbay, Opt. Lett. 26, 2623 (2004). 